\title{
Deep vein thrombosis of the upper extremity caused by central venous port in a patient with soft tissue sarcoma: A case report
}

\author{
KOUJI KITA, TOMOKI NAKAMURA, KOUICHI NAKAMURA, \\ TOMOHITO HAGI, KUNIHIRO ASANUMA and AKIHIRO SUDO
}

\author{
Department of Orthopedic Surgery, Mie University Graduate School of Medicine, Tsu, Mie 514-8507, Japan
}

Received December 10, 2019; Accepted July 29, 2020

DOI: $10.3892 / \mathrm{mco} .2020 .2160$

\begin{abstract}
Deep vein thrombosis (DVT) more commonly occurs in the lower extremities, whereas involvement of the upper extremities is rare. The present case report describes the clinical course of the development and treatment of upper extremity DVT (UEDVT) following insertion of an indwelling central venous $(\mathrm{CV})$ port in a patient with soft tissue sarcoma (STS) of the thigh. A 66-year-old man was referred to our hospital for STS treatment. The indwelling CV port was placed via the left subclavian vein, and two courses of neoadjuvant chemotherapy were administered. Two months after the catheter placement, DVT was detected from the left upper arm to the left internal jugular vein. Anticoagulation therapy with warfarin was started and DVT was undetectable at 5 months after surgery. In conclusion, DVT may occur in cancer patients who undergo treatment with indwelling CV ports. Therefore, screening should be conducted concurrently with surgical resection and chemotherapy for STS.
\end{abstract}

\section{Introduction}

Patients with cancer are at high risk of developing deep vein thrombosis (DVT), which occurs mostly in the lower extremities and rarely in the upper extremities (1). Approximately $4-10 \%$ of all cases of DVT involve the subclavian, axillary, or brachial veins. The use of a central venous catheter (CVC) improves the management of patients with cancer. However, the presence of CVC increases the risk of developing upper extremity DVT (UEDVT) and its associated complications, such as pulmonary embolism (PE) (1). Although development of DVT after placement of an indwelling CVC has been commonly reported (2), there are few reports of DVT occurring

Correspondence to: Dr Tomoki Nakamura, Department of Orthopedic Surgery, Mie University Graduate School of Medicine, 2-174 Edobashi, Tsu, Mie 514-8507, Japan

E-mail: tomoki66@clin.medic.mie-u.ac.jp

Key words: deep vein thrombosis, upper extremity, central venous port after the placement of an indwelling CV port in patients with soft tissue sarcoma (STS).

For the effective management of high-grade STS, surgical tumor resection combined with neoadjuvant chemotherapy is recommended and the placement of an indwelling $\mathrm{CV}$ port may also be considered (3). We herein report the clinical course of a patient with STS of the thigh who developed UEDVT following indwelling CV port placement.

\section{Case report}

A 66-year-old man visited a local clinic in November 2012, 1 month after noticing a mass developing in his right thigh. The patient was referred to the Department of Orthopedic Surgery at Mie University Hospital on suspicion of STS. On physical examination, a swelling was identified on the lateral side of the right thigh. There was no fever, tenderness, or redness.

Magnetic resonance imaging examination revealed a soft tissue mass that involved the vastus lateralis and vastus intermedius muscles, and exhibited low signal intensity on T1-weighted images and heterogenous signal intensity on T2-weighted images; the administration of gadolinium enhanced the heterogeneous signal intensity (Fig. 1).

Plain and contrast-enhanced computed tomography (CT) examination of the chest, abdomen and pelvis did not demonstrate any distant metastasis.

Further examination of a Tru-Cut biopsy specimen revealed undifferentiated pleomorphic sarcoma. Subsequently, an indwelling CV port was placed through the left subclavian vein and the patient underwent two courses of neoadjuvant chemotherapy using doxorubicin $\left(60 \mathrm{mg} / \mathrm{m}^{2}\right)$ and ifosfamide $\left(10 \mathrm{~g} / \mathrm{m}^{2}\right)$, as the tumor was high-grade, deeply situated, and large. Two months after the indwelling port placement, a preoperative contrast-enhanced CT scan of the chest at the screening of post neo-adjuvant chemotherapy revealed DVT of the left internal jugular vein. Therefore, contrast-enhanced CT of brain, neck and upper extremity were further performed and DVT from the left upper arm to the left internal jugular vein was observed (Fig. 2) The D-dimer level was $8.63 \mu \mathrm{g} / \mathrm{ml}$ (standard, $<1.0 \mu \mathrm{g} / \mathrm{ml}$ ).

There were no associated symptoms, such as swelling or pain, and PE was not detected on the CT images. After administering anticoagulation therapy with heparin (10,000 U/day), wide tumor resection and reconstruction using a prosthesis 

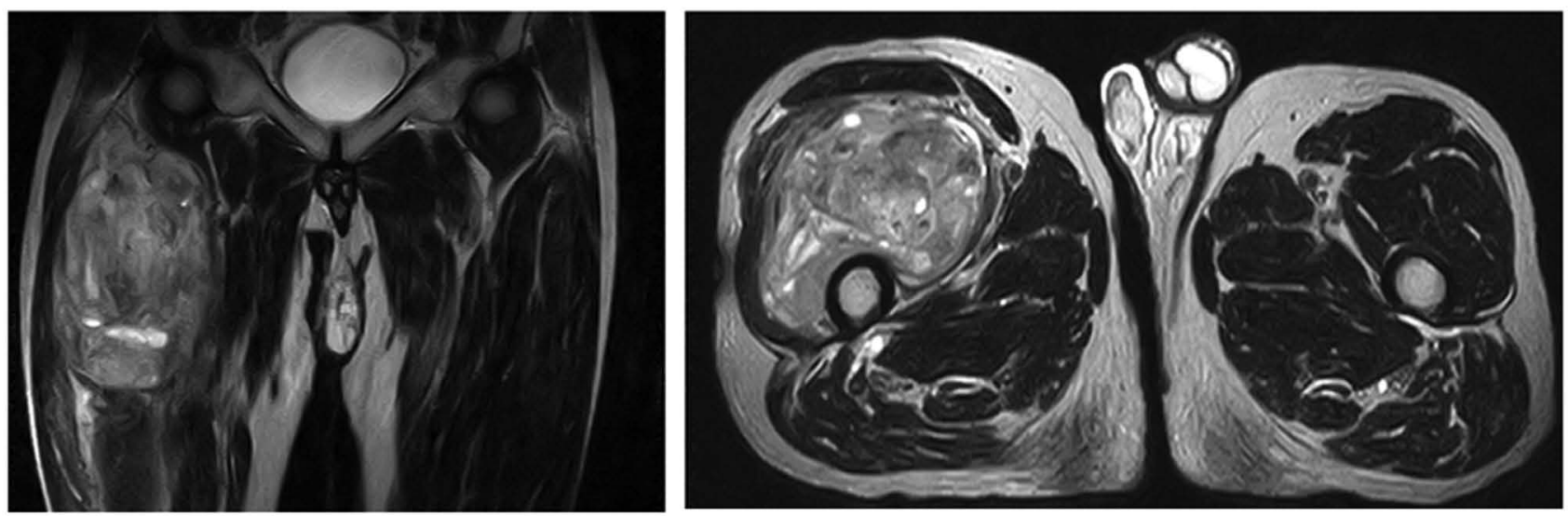

Figure 1. A soft tissue mass inside the vastus lateralis and vastus intermedius muscles observed on magnetic resonance imaging. The administration of gadolinium enhanced heterogeneous signal intensity (right, coronal view; left, axial view).
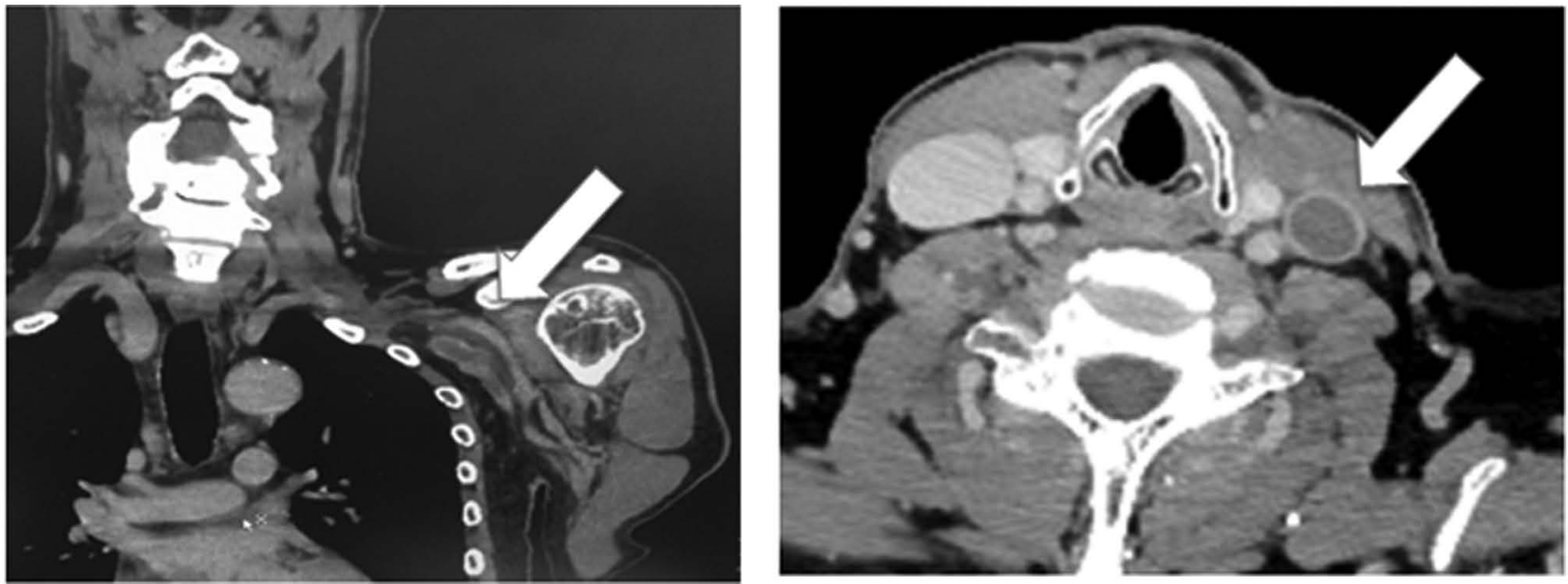

Figure 2. Contrast-enhanced computed tomography scan showing the deep vein thrombosis extending from the left upper arm to the left internal jugular vein (arrow).

were performed. One week after the surgery, anticoagulation therapy with heparin was resumed; however, it was discontinued due to the progression of anemia (hemoglobin level $6.1 \mathrm{~g} / \mathrm{dl}$; normal range, $13.5-16.8 \mathrm{~g} / \mathrm{dl}$ ) and a blood transfusion was performed. After 1 week, anticoagulation therapy with warfarin was resumed, as the findings on contrast-enhanced CT revealed an increase in thrombosis. There were no complications during the treatment.

On the 5-month postoperative contrast CT scan, DVT was not detected and the indwelling $\mathrm{CV}$ port was removed due to the risk of thrombosis (Fig. 3). The D-dimer levels had decreased to $3.28 \mu \mathrm{g} / \mathrm{ml}$. Furthermore, warfarin was discontinued 1 month after the port removal. Four months later, the patient developed local recurrence of STS in the right thigh that was treated with wide resection and endoprosthesis-based reconstruction. Although adjuvant radiotherapy was performed, it was discontinued due to the development of a postoperative infection (pathogen: Serratia marcescens). Finally, debridement and revision surgery were performed; however, the patient developed multiple metastases and eventually succumbed to the disease on May 8th, 2015.

\section{Discussion}

Patients with cancer are at a high risk of developing DVT. Piccioli et al reported that cancer cells can cause endothelial cell injury, thereby intensifying hypercoagulability (4). A CVC is commonly used in cancer patients who require chemotherapy and intravenous administration of supportive treatment, such as antiemetic and diuretic drugs, although its presence increases the risk of DVT (2). However, it was decided to proceed with the $\mathrm{CV}$ port placement in the present case, as the sarcoma was large and high-grade, requiring neoadjuvant chemotherapy and wide resection (3).

During the administration and clinical course of chemotherapy in cancer patients with an indwelling CV port, DVT is typically triggered when a coating of clotted blood and blood proteins forms around the catheter (5).

Marinella et al reported that, among 90 patients who developed UEDVT, the most common underlying conditions were the presence of CVC in 65 patients $(72 \%)$, infection in $25(28 \%)$, extrathoracic malignancy in $20(22 \%)$, thoracic malignancy in $19(21 \%)$, and a prior lower extremity DVT in 16 cases (18\%) (6). 

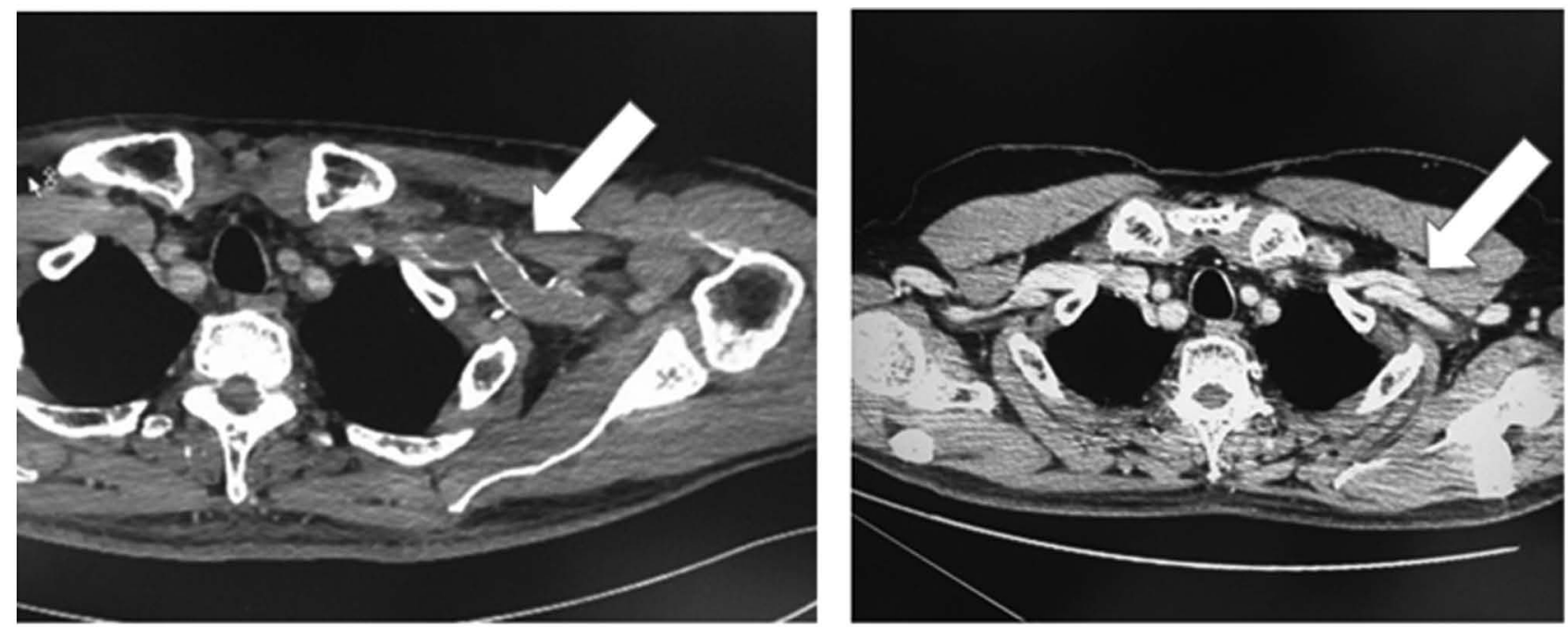

Figure 3. Computed tomography image prior to the anticoagulation therapy showing the DVT (left, arrow). DVT had disappeared after 5 months of administering anticoagulant therapy (right, arrow). DVT, deep vein thrombosis.

The onset of UEDVT is usually characterized by arm swelling, edema and pain, but completely asymptomatic cases are possible, particularly in patients with long-term CVC placement $(6,7)$. In the present case, there were no aforementioned symptoms, and DVT was detected incidentally on preoperative contrast CT.

In UEDVT, early diagnosis is crucial, even for asymptomatic cases, due to the risk of PE that may occur due to catheter-related thrombosis. It was previously reported that $\mathrm{PE}$ was implicated in $\sim 10 \%$ of UEDVT cases (8). Recently, a lower rate $(5 \%)$ of $\mathrm{PE}$ in patients with isolated catheter-associated UEDVT has been documented $(9,10)$. Jones et al also described that anticoagulation may not affect the rate of resolution or decrease the progression of UEVDT, whereas it is associated with a significant incidence of bleeding complications (9). Therefore, there is currently no consensus on the optimal management of UEDVT.

Early detection may be difficult when there are no symptoms, such as swelling or pain. Marinella et al noted pain and edema in 34 and $84 \%$ of the UEDVT cases, respectively (6). In a study by Hylton et al, swelling was observed in $82 \%$ of the patients who developed UEDVT after CVC insertion, but no symptoms were present in $6 \%$ of the cases (7). DVT may be detected by contrast CT and ultrasonography and, upon confirmation of UEDVT, anticoagulant therapy may be administered. To the best of our knowledge, the present report is the first to describe in detail the clinical course of UEDVT in a sarcoma patient with an indwelling CV port. Although sarcoma is rare and fewer patients with sarcoma receive chemotherapy compared to those with cancer, the possibility of UEDVT and necessity of screening sarcoma patients with indwelling CV ports should be considered. There was a limitation to the present case report: Ultrasound examination was not performed, as the possibility of UEDVT was not taken into consideration prior to its detection on CT scan. In conclusion, we herein report the case of a patient with UEDVT who was asymptomatic. Therefore, even in the absence of any symptoms, we recommend that screening for DVT should be performed in patients with indwelling CV ports.

\section{Acknowledgements}

Not applicable.

\section{Funding}

No funding was received.

\section{Availability of materials and data}

Not applicable.

\section{Authors' contributions}

TN conceived the study, treated the patient, collected the data and wrote the manuscript. KK collected, analyzed and interpreted the clinical data. KA and TH performed the surgery, and analyzed and interpreted the clinical data. KN collected and analyzed the clinical data. AS analyzed and interpreted the clinical data, and reviewed the manuscript.

\section{Ethics approval and consent to participate}

Not applicable.

\section{Patient consent for publication}

Written informed consent was obtained from the patient for the publication of the case details and associated images.

\section{Competing interests}

The authors declare that they have no competing interests.

\section{References}

1. Gaddh M, Antun A, Yamada K, Gupta P, Tran H, EI Rassi F, Kim HS and Khoury HJ: Venous access catheter-related thrombosis in patients with cancer. Leuk Lymphoma 55: 501-508, 2018. 
2. Yukisawa S, Fujiwara Y, Yamamoto Y, Ueno T, Matsueda K, Kohno A and Suenaga M: Upper-extremity deep vein thrombosis related to central venous port systems implanted in cancer patients. Br J Radiol 83: 850-853, 2010.

3. Tanaka K, Kawamoto H, Saito I, Yoshimura K, Fukuda H and Iwamoto Y: Preoperative and postoperative chemotherapy with ifosfamide and adriamycin for adult high-grade soft-tissue sarcomas in the extremities: Japan clinical oncology group study JCOG0304. Jpn J Clin Oncol 39: 271-273, 2009.

4. Ay C, Pabinger I and Cohen AT: Cancer associated venous thromboembolism: Burden, mechanisms, and management. Thromb Haemost 117: 219-230, 2017.

5. Evans NS and Ratchford EV: Catheter-related venous thrombosis. Vasc Med 23: 411-413, 2018.

6. Marinella MA, Kathula SK and Markert RJ: Spectrum of upper-extremity deep venous thrombosis in a community teaching hospital. Heart Lung 29: 113-117, 2000.

7. Joffe HV, Kucher N, Tapson VF and Goldhaber SZ: Deep Vein Thrombosis (DVT) FREE Steering Committee: Upper-extremity deep vein thrombosis: A prospective registry of 592 patients. Circulation 110: 1605-1611, 2004.
8. Verso $\mathrm{M}$ and Agnelli G: Venous thromboembolism associated with long-term use of central venous catheters in cancer patients. J Clin Oncol 21: 3665-3675, 2003

9. Jones MA, Lee DY, Segall JA, Landry GJ, Liem TK, Mitchell EL and Moneta GL: Characterizing resolution of catheter-associated upper extremity deep venous thrombosis. J Vasc Surg 51: 108-113, 2020.

10. Ploton G, Pistorius MA, Raimbeau A, Denis Le Seve J, Bergère G, Ngohou C, Goueffic Y, Artifoni M, Durant C, Gautier G, et al: A STROBE cohort study of 755 deep and superficial upper-extremity vein thrombosis. Medicine (Baltimore) 99: e18996, 2020. 